\title{
Severe diabetic ketoacidosis complicated by hypocapnic seizure
}

\author{
A Majid' and B J Wheeler1,2 \\ 1Paediatric Endocrinology, Southern District Health Board, Dunedin, New Zealand and 2Department of \\ Women's and Children's Health, University of Otago, Dunedin School of Medicine, Dunedin, New Zealand
}

Correspondence should be addressed to B J Wheeler

Email

ben.wheeler@otago.ac.nz

\section{Summary}

In clinical practice, seizures independent of hypoglycemia are observed in patients with type 1 diabetes mellitus (T1DM) more frequently than expected by chance, suggesting a link. However, seizures during management of diabetic ketoacidosis (DKA) have generally been considered a bad prognostic factor, and usually associated with well-known biochemical or neurological complications. We present the case of a 17-year-old girl with known T1DM managed for severe DKA complicated by hypocapnic seizure. We review the literature on this rare occurrence as well as outline other possible differentials to consider when faced with the alarming combination of DKA and seizure.

\section{Learning points:}

- Seizures during DKA treatment require immediate management as well as evaluation to determine their underlying cause.

- Their etiology is varied, but a lowered seizure threshold, electrolyte disturbances and serious neurological complications of DKA such as cerebral edema must all be considered.

- Sudden severe hypocapnia may represent a rare contributor to seizure during the treatment of DKA.

\section{Background}

Diabetic ketoacidosis (DKA) is an endocrine emergency occurring in patients with both new-onset and established type 1 diabetes (T1DM). In brief, the diagnosis is based on clinical suspicion followed by fulfillment of biochemical criteria: Hyperglycemia (blood glucose level (BGL) $>11 \mathrm{mmol} / \mathrm{L}$ ); ketonuria and/or ketonemia and metabolic acidosis ( $\mathrm{pH}<7.3$, bicarbonate $<15 \mathrm{mmol} / \mathrm{L}$ ).

It is a leading acute cause of diabetes-related morbidity and mortality, particularly in children (1), often due to well-described complications of treatment. The most common of these include cerebral edema, and electrolyte disturbances such as hypoglycemia and hypokalemia.

Seizure activity occurring during treatment of DKA is therefore a cause for considerable alarm due to the possibility it may represent a symptom of one of these iatrogenic complications. We present the case of an adolescent girl with severe DKA complicated by subsequent seizure, and use this as an opportunity to review the literature on this subject and discuss possible differentials. The likely causes in this case being severe hypocapnia combined with a lowered seizure threshold, a combination not previously described in childhood DKA.

\section{Case presentation}

A 17-year-old girl with established T1DM presented with a one-day history of lethargy, vomiting and abdominal pain. She had been discharged from 
hospital the previous day following resolution of symptoms of possible appendicitis, which had led to removal of a non-inflamed appendix. During this prior surgical admission, normal biochemistry was regularly monitored, including: BGL: $9.5 \mathrm{mmol} / \mathrm{L}$ (3.5-7.5), serum ketones: $0.1 \mathrm{mmol} / \mathrm{L}$, venous blood gas $\mathrm{pH}: 7.42$ (7.35-7.45), $\mathrm{pCO}_{2}: 36 \mathrm{mmHg}(35-45), \mathrm{pO}_{2}: 52 \mathrm{mmHg}$ (30-40), $\mathrm{HCO}_{3}: 23.1 \mathrm{mmol} / \mathrm{L}(22.0-32.0)$, base excess: $-0.4 \mathrm{mmol} / \mathrm{L}(-2.0$ to 2.0$)$, lactate: $5.4 \mathrm{mmol} / \mathrm{L}(0.5-2.2)$ (all excluding DKA/euglycaemic DKA), serum sodium: $138 \mathrm{mmol} / \mathrm{L}$ (135-145) and potassium: $4.1 \mathrm{mmol} / \mathrm{L}$ (3.5-5.2). However, while her BGLs were stable during this surgical admission, the subsequent change to her routines had led to complete insulin omission for at least $24 \mathrm{~h}$ prior to representation. Her clinical and biochemical presentation was consistent with severe DKA: BGL: $22.4 \mathrm{mmol} / \mathrm{L}$; serum ketones: $4.6 \mathrm{mmol} / \mathrm{L}$ $(<0.6)$; venous blood gas showed $\mathrm{pH}: 6.99$ (7.35-7.45), $\mathrm{pCO}_{2}$ : $33 \mathrm{mmHg}(35-45), \mathrm{pO}_{2} 32 \mathrm{mmHg}$ : (30-40), $\mathrm{HCO}_{3}$ : $7.4 \mathrm{mmol} / \mathrm{L}$ (22.0-32.0), base excess: $-24.6 \mathrm{mmol} / \mathrm{L}(-2.0$ to 2.0 ), and lactate: $2.8 \mathrm{mmol} / \mathrm{L}(0.5-2.2)$. Electrolytes at the time of presentation: corrected sodium: $132 \mathrm{mmol} / \mathrm{L}$ (135-145), serum calcium: $2.5 \mathrm{mmol} / \mathrm{L}$ (2.2-2.6), ionized calcium: $1.27 \mathrm{mmol} / \mathrm{L}$ (1.1-1.35), serum phosphate: $1.8 \mathrm{mmol} / \mathrm{L}(0.8-1.5)$ while serum magnesium could not be obtained on presentation as the sample hemolyzed. On evaluation in ED the patient was oriented in time, place and person but was lethargic. She had cool peripheries and brisk central refill time of less than $2 \mathrm{~s}$ and had dry mucous membranes. Her pupils were bilaterally equal and reactive and she was tachypneic with a respiratory rate of 32 . Her abdomen was soft, nondistended but had mild, generalized tenderness. She was commenced on therapy consistent with the New Zealand National DKA guidelines for children (available from: https://www.starship.org.nz/media/273118/starshipdka-guideline-2014.pdf).

Two and half hours into treatment (80 min after insulin infusion), she became unresponsive, and had a generalized tonic-clonic convulsion lasting $3 \mathrm{~min}$. This was the first seizure she had ever experienced. Following this episode her prominent Kussmaul breathing persisted, but neurological observations remained within normal limits, her pupils were reactive, her oxygen saturations were $100 \%$ in room air, she was normotensive and had $<2$-s central capillary return. She was transferred from high-dependency level care (HDU) to the intensive care unit (ICU) for continuation of care, and for further evaluation and management of possible cerebral edema.

\section{Investigation}

As mentioned previously, her initial blood tests were consistent with a diagnosis of severe DKA, and repeat bloods immediately following her seizure revealed further deterioration: arterial blood gas (in air): $\mathrm{pH}: 6.92$ (7.35-7.45), $\mathrm{pCO}_{2}$ : $7 \mathrm{mmHg}$ (35-45); $\mathrm{pO}_{2}: 173 \mathrm{mmHg}$ (80-100), $\mathrm{HCO}_{3}: 1.4 \mathrm{mmol} / \mathrm{L}$ (22.0-32.0); base excess: $-25.0 \mathrm{mmol} / \mathrm{L}(-2.0$ to 2.0$)$ and lactate: $2.1 \mathrm{mmol} / \mathrm{L}$ (0.5-2.2). Basic electrolytes were largely unchanged with a BGL of $21 \mathrm{mmol} / \mathrm{L}$; serum ketones: $4.4 \mathrm{mmol} / \mathrm{L}$; corrected sodium: $138.7 \mathrm{mmol} / \mathrm{L}$; serum total calcium: $2.5 \mathrm{mmol} / \mathrm{L}$ (2.2-2.6); ionized calcium: $1.27 \mathrm{mmol} / \mathrm{L}$ (1.11.35); serum phosphate: $1.7 \mathrm{mmol} / \mathrm{L}(0.8-1.5)$ and serum $\mathrm{Mg}$ : $0.9 \mathrm{mmol} / \mathrm{L}(0.6-1.2)$. She underwent an urgent computed tomography (CT) head scan that showed no evidence of cerebral edema and venous sinus thrombosis. A subsequent neurology opinion was sought, and based on the single seizure, no past or family history and stable neurological/cognitive status, no further investigations (such as EEG or MRI) were recommended.

\section{Treatment}

In brief, her initial treatment consisted of a $10 \mathrm{~mL} / \mathrm{kg}$ bolus of $0.9 \%$ saline, followed by intravenous $0.9 \%$ saline, rate determined by her level of dehydration replaced over $48 \mathrm{~h}$ and daily maintenance. Insulin therapy with intravenous Actrapid was delayed (as per protocol above) and then commenced at $0.1 \mathrm{unit} / \mathrm{kg} / \mathrm{h} 80 \mathrm{~min}$ from fluid therapy commencement.

Following her seizure, she was admitted to the ICU. Consideration was given for immediate hypertonic saline for possible cerebral edema, but due to her stable state post seizure, the early onset in relation to treatment and the immediate availability of CT scanning, this was deferred. Over the course of her admission, her DKA resolved and she did not have any further seizures. She also had surgical consults with the general and gynecology surgical sub-specialties and was cleared of any further surgical pathology by these assessments and a CT scan of her abdomen.

\section{Outcome and follow-up}

The following day she was much improved, and after a further day in hospital, she was discharged home on her standard subcutaneous insulin regimen. She continues with regular clinic follow-up and now 3 months following this episode, she has not had any further seizures. 
Table 1 Differential diagnoses for seizures during treatment of diabetic ketoacidosis.

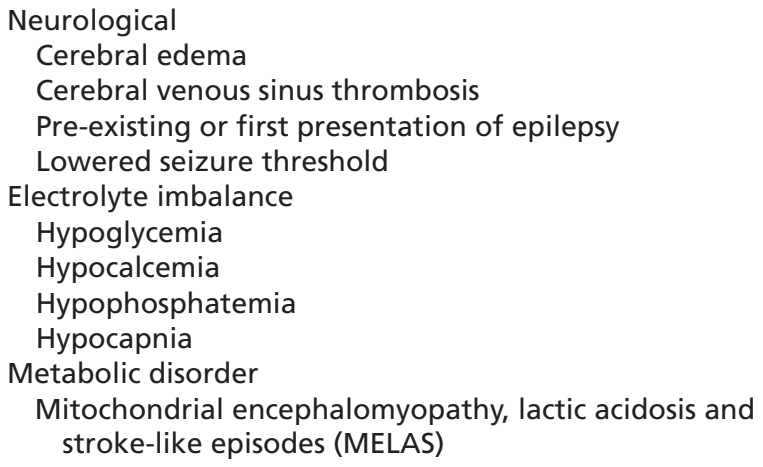

\section{Discussion}

Seizures during treatment for DKA are rarely seen, nor described in the medical literature. Possible differential diagnoses are highlighted in Table 1.

In this case, in the absence of any other clear explanation, we propose that based on the spontaneous and sustained clinical improvement, minimally deranged electrolytes and normal scans, her convulsion was primarily caused by severe hypocapnia $\left(\mathrm{PaCO}_{2}\right.$ of $\left.7 \mathrm{mmHg}\right)$. Kussmaul breathing associated with hyperventilation is a characteristic feature of diabetes ketoacidosis, and resultant hypocapnia is a well-described phenomenon resulting from respiratory compensation of marked metabolic acidosis. In this case, given the very low $\mathrm{pCO}_{2}$, additional hyperventilation over and above respiratory compensation is also likely implicated, and while a component of cerebral edema cannot be completely excluded, a contribution from fear and anxiety would also be reasonable assumptions. While seizure attributed to hypocapnia has not been previously described in the diabetes literature, it has been identified in the wider scientific literature (2). The proposed mechanism being hyperventilation (from whatever cause) and resultant hypocapnia leading to cerebral vasoconstriction and decreased cerebral blood flow (3).

In addition, possibly also contributing to this presentation is a diabetes-related lowered seizure threshold, particularly in the context of severe illness and acidosis. A number of studies have explored possible links between seizures and T1DM. While some have been inconclusive (4), and none have specifically dealt with DKA, seizures and TIDM appear to be associated, especially in children. Recently Ramakrishnan and coworkers suggested that 2.1\% of children with TIDM in their United Kingdom dataset had seizures, which is approximately six times greater than the prevalence of epilepsy in the general population (5). Diabetes-related antibodies may also play a role, with glutamic acid decarboxylase (GAD) a major autoantigen in TIDM implicated in patients with seizures (6).

Arguably, the most important alternative diagnosis for seizure in the DKA context is cerebral edema, the most feared and leading cause of DKA treatment related morbidity and mortality (1). Clinically significant cerebral edema occurs in approximately one percent of DKA episodes in children and has a reported mortality rate of $20-90 \%(1,7)$. Cerebral edema is most likely to occur between 4 and $12 \mathrm{~h}$ after the initiation of therapy, but may occur at other times, rarely even prior to initiation of therapy or after the first $24 \mathrm{~h}$ (8). While unlikely as a key cause in the case described, due to the stable clinical progress both prior and after the seizure, the early onset of seizure and normal brain imaging, this remains the key diagnosis to exclude and/or empirically treat when scenarios like these are encountered. Cerebral venous thrombosis is the other neurological complication of DKA potentially causing seizure. Cerebral venous sinus thrombosis is a condition with wide spectrum of clinical symptoms and signs and the occurrence is rare in DKA cases, the primary mechanism being dehydration and a prothrombotic state associated with DKA (9). While a well-described cause of morbidity in DKA, only a single case report has been published highlighting this as a cause of seizure (10).

A range of biochemical derangements can also lead to seizure during DKA management. Firstly, as the brain uses glucose as a primary fuel for energy generation, it is no surprise that seizures can be a consequence of severe hypoglycemia. This remains one of the most feared acute complications of T1DM, and hypoglycemic seizures are well described (11). This was excluded in our case as seizure occurred after only $120 \mathrm{~min}$ of treatment while BGLs were clearly still in the hyperglycemic range $(22.1 \mathrm{mmol} / \mathrm{L})$. Reduced ionized calcium is also a pertinent cause of seizures in children without diabetes. In diabetes, hypocalcemia may be related to hypomagnesemia, by impaired release of PTH or skeletal or renal tubule resistance to action of PTH (12). In DKA, hypocalcemia is poorly explained and symptomatic presentations with seizures are rarely described. Again, based on normal calcium status immediately post seizure, and throughout admission, this was excluded as a likely cause. Finally, hypophosphatemia has also been infrequently associated with seizure and DKA, but only once in a child (13). 
In conclusion, we present a rare case of DKA complicated by likely hypocapnic seizure. A lowered seizure threshold may have also played an important adjunctive role. This case report provides a timely opportunity to bring together the diverse literature on this topic and highlights the potential differentials to consider when confronted by this alarming combination.

\section{Patient's perspective}

We welcome comments from your patient; their own description of their experience may help other patients or clinicians who are dealing with a similar problem. If your patient would like to contribute please ensure they include only relevant personal details. Patients may describe their symptoms, how any tests and treatments affected them and how the problem is now.

\section{Declaration of interest}

The authors declare that there is no conflict of interest that could be perceived as prejudicing the impartiality of the research reported.

\section{Funding}

This research did not receive any specific grant from any funding agency in the public, commercial or not-for-profit sector.

\section{Patient consent}

Written informed consent has been obtained from the patient (and the patient's guardian) for publication of this article.

\section{Author contribution statement}

A Majid and B J Wheeler provided clinical care to the patient. A Majid produced the first draft of the manuscript and along with B J Wheeler coordinated the manuscript through subsequent editing and submission. B J Wheeler conceived and supervised the manuscript. Both authors reviewed and approved the final version.

\section{References}

1 Edge JA, Hawkins MM, Winter DL \& Dunger DB 2001 The risk and outcome of cerebral oedema developing during diabetic ketoacidosis. Archives of Disease in Childhood 85 16-22. (doi:10.1136/adc.85.1.16)

2 Holmes MD, Dewaraja AS \& Vanhatalo S 2004 Does hyperventilation elicit epileptic seizures? Epilepsia 45 618-620. (doi:10.1111/ j.0013-9580.2004.63803.x)

3 Raichle ME \& Plum F 1972 Hyperventilation and cerebral blood flow. Stroke 3 566-575. (doi:10.1161/01.STR.3.5.566)

4 O'Connell MA, Harvey AS, Mackay MT \& Cameron FJ 2008 Does epilepsy occur more frequently in children with Type 1 diabetes? Journal of Paediatrics and Child Health 44 586-589. (doi:10.1111/ j.1440-1754.2008.01387.x)

5 Ramakrishnan R \& Appleton R 2012 Study of prevalence of epilepsy in children with type 1 diabetes mellitus. Seizure 21 292-294. (doi:10.1016/j.seizure.2012.01.003)

6 Peltola J, Kulmala P, Isojarvi J, Saiz A, Latvala K, Palmio J, Savola K, Knip M, Keränen T \& Graus F 2000 Autoantibodies to glutamic acid decarboxylase in patients with therapy-resistant epilepsy. Neurology $\mathbf{5 5}$ 46-50. (doi:10.1212/WNL.55.1.46)

7 Schober E, Otto KP, Dost A, Jorch N \& Holl R 2012 Association of epilepsy and type 1 diabetes mellitus in children and adolescents: is there an increased risk for diabetic ketoacidosis? Journal of Pediatrics 160 662.e1-666.e1. (doi:10.1016/j.jpeds.2011.09.054)

8 Dunger DB, Sperling MA, Acerini CL, Bohn DJ, Daneman D, Danne TP, Glaser NS, Hanas R, Hintz RL, Levitsky LL, et al. 2004 ESPE/ LWPES consensus statement on diabetic ketoacidosis in children and adolescents. Archives of Disease in Childhood 89 188-194. (doi:10.1136/adc.2003.044875)

9 Carl GF, Hoffman WH, Passmore GG, Truemper EJ, Lightsey AL, Cornwell PE, \& Jonah MH 2003 Diabetic ketoacidosis promotes a prothrombotic state. Endocrine Research 29 73-82. (doi:10.1081/ERC-120018678)

10 Keane S, Gallagher A, Ackroyd S, McShane MA \& Edge JA 2002 Cerebral venous thrombosis during diabetic ketoacidosis. Archives of Disease in Childhood 86 204-205. (doi:10.1136/adc.86.3.204)

11 Bulsara MK, Holman CD, Davis EA \& Jones TW 2004 The impact of a decade of changing treatment on rates of severe hypoglycemia in a population-based cohort of children with type 1 diabetes. Diabetes Care 27 2293-2298. (doi:10.2337/diacare.27.10.2293)

12 Liamis G, Milionis HJ \& Elisaf M 2009 A review of drug-induced hypocalcemia. Journal of Bone and Mineral Metabolism 27 635-642. (doi:10.1007/s00774-009-0119-x)

13 de Oliveira Iglesias SB, Pons Leite H \& de Carvalho WB 2009 Hypophosphatemia-induced seizure in a child with diabetic ketoacidosis. Pediatric Emergency Care 25 859-861. (doi:10.1097/ PEC.0b013e3181c399f6)

Received in final form 2 May 2017

Accepted 6 June 2017 